\title{
The Impact of COVID-19 on Cancer
}

\section{Yue Li \\ Xingjian Wang \\ Wei Wang}

Department of Respiratory and Critical Care Medicine, The First Hospital of China Medical University, Shenyang, People's Republic of China
Correspondence: Wei Wang

Department of Respiratory and Critical Care Medicine, The First Hospital of

China Medical University, I 55 Nanjing

North Street, Heping District, Shenyang,

Liaoning, II000I, People's Republic of

China

Email wwbycmu@I26.com

\begin{abstract}
Since late December 2019, the 2019 coronavirus disease (COVID-19) caused by severe acute respiratory syndrome coronavirus 2 (SARS-CoV-2) and its rapid international spread have posed a global health threat. The World Health Organization has declared the outbreak of COVID-19 as "public health emergency of international concern". COVID-19 not only brings tremendous pressure to the medical system but also brings new challenges to the global economy. The occurrence and development of cancer has always been an area of active research, and COVID-19 also has a long-lasting impact on the diagnosis, treatment, and research of cancer. In the context, we review the adverse effects of COVID-19 on the screening, diagnosis, treatment and prognosis of cancer patients and the countermeasures in this situation, and provide solutions for improving the quality of life of cancer patients in the normalized prevention and control of COVID-19.
\end{abstract}

Keywords: COVID-19, SARS-CoV-2, cancer

\section{Introduction}

Severe Acute Respiratory Syndrome Coronavirus (SARS-CoV)-2 (SARS-CoV-2) is a new type of coronavirus belonging to the same $\beta$-coronavirus genus as SARS$\mathrm{CoV}$ and Middle East Respiratory Syndrome Coronavirus (MERS-CoV). ${ }^{1}$ Coronavirus disease 2019 (COVID-19) caused by SARS-CoV-2 infection has spread worldwide. According to an investigation on 2878 patients with COVID19 conducted by Prof. Nanshan Zhong's team, fever (83.0\%) was the most common symptom, and $63.4 \%$ of the patients presented fever as onset symptom. ${ }^{2}$ Besides serious respiratory symptoms, COVID-19 also causes several extrapulmonary manifestations, such as thrombosis complications, myocardial dysfunction and arrhythmia, acute coronary syndrome, acute kidney injury, gastrointestinal symptoms, liver cell injury, hyperglycemia and ketosis, neurological diseases, ocular symptoms, and dermatological complications. ${ }^{3}$ It shows flu-like symptoms with high contagion rate and severe pathogenicity. COVID-19 has been included in the Law of the People's Republic of China on the Prevention and Control of Infectious Diseases as a Class $\mathrm{B}$ infectious disease, and preventive and control measures for Class A infectious diseases have been adopted. ${ }^{4}$ As of January 5, 2021, the total number of confirmed cases worldwide is about 83.33 million, and the cumulative number of deaths is about 1.83 million. ${ }^{5}$ It has brought a heavy burden to the public health system, which not only severely retarded social development but also affected the diagnosis and treatment of other diseases. Cancer is a complex group of diseases whose prognosis is affected by the timely diagnosis, treatment, and intervention. For one thing, due to the existence of COVID-19, the diagnosis and treatment of cancer is delayed and the condition of cancer is deteriorated. Further, due to the 
immunosuppressive status of cancer patients, they are more susceptible to SARS-CoV-2 infection, and reciprocally, SARS-CoV-2 increases the poor prognosis of patients with cancer. ${ }^{6}$ The Lancet Oncology calls for great attention to the diagnosis and treatment of cancer during the normalized prevention and control of COVID$19,^{7}$ and emphasizes the need to maximize the treatment benefits of patients with cancer under this background. To address these issues, we summarize the research on cancer and COVID-19 to clarify the impact of COVID-19 on cancer.

\section{The Impact of Covid-I 9 on Cancer Diagnosis and Treatment}

The shortage and busyness of medical staff due to the admission of a large number of COVID-19 patients have reduced the interaction frequency between patients and oncologists by $20 \%$ in the United States, which postpones more than 22 million malignant tumor screening tests. ${ }^{8,9}$ Currently, the challenges faced by oncology nursing staff include shortage of manpower, lack of specialized training, occupational safety issues (including the impact of COVID-19) and burnout. The European Society of Medical Oncology (ESMO) investigated the COVID-19 pandemic's impact on the burnout, work performance and health status of global oncology workers. In the first survey conducted from April 16 to May 3, 2020, there were 1520 participants from 101 countries. $38 \%$ of respondents reported the feeling of burnout while $78 \%$ of respondents were increasingly concerned about their personal safety since the onset of the pandemic. More seriously, in the second survey conducted from July 16 to August 6, 2020, the proportion of respondents who reported burnout has risen to $49 \%{ }^{10}$ Bakouny et al's research further showed that during the COVID-19 pandemic, the number of patients undergoing cancer screening and the number of those who subsequently diagnosed with cancers or precancerous lesions both decreased significantly in Brigham, Massachusetts, that is the largest health care system in the northeastern United States. ${ }^{11}$ Routine physical examination of the human body plays an important role in the early diagnosis of various cancers. ${ }^{12}$ On March 16, 2020, the United Kingdom postponed routine screening for cancer in response to the COVID-19 pandemic. According to Maringe et al's estimation of the postponement's influence on cancer diagnosis, compared with the data before the pandemic, the number of deaths caused by breast, colorectal, lung, and esophageal cancer will increase by $7.9-9.6 \%, 15.3-16.6 \%, 4.8-5.3 \%$, and $5.8-6.0 \%$ in five years, respectively. ${ }^{13}$ Measures are adopted in many hospitals to limit the number of outpatients and inpatients. For cancer patients with stable disease, only emergency surgery could be performed in time but adjuvant chemotherapy or elective surgery may be postponed to reduce the risk of cross-infection. ${ }^{14}$ The compulsory sudden delay or interruption of conventional treatment may increase the risk of deterioration in patients with cancer and reduce their survival rates. ${ }^{15}$ Sud et al reported that a six-month delay in malignant tumor surgery will cause the life-years gained drop from 18.1 to $15.9{ }^{16}$ Riemann et al followed up 1080 patients after Ear Nose Throat (ENT) surgery for 16 weeks and found that due to the impact of COVID-19 pandemic, the diagnostic procedures for suspected cancer were reduced by $41 \%$, and the critical procedures for surgery were reduced by $43 \%$; at the same time, due to the cancellation of elective surgery, the proportion of emergency operations has increased by $90 \% .{ }^{17}$ Besides, some anti-cancer agents that require infusion had to be changed to oral delivery during COVID-19 pandemic, which affected their therapeutic effect. Zhang et al conducted a retrospective cohort study of COVID-19 laboratory-confirmed cancer patients in three designated hospitals in Wuhan, China, and found that within 14 days, anti-cancer treatment was significantly related to the occurrence of serious clinical events in COVID-19 infection (In this study, a serious event was defined as admission to an ICU, requiring mechanical ventilation, or death). ${ }^{18}$ Therefore, some oncologists and societies recommend that before the symptoms of COVID-19 have completely disappeared and the virus test is negative, chemistry should generally not be started treatment. ${ }^{19,20}$ Second, the implementation of immunosuppressive chemotherapy in recovered COVID-19 patients may lead to disease recurrence. Lancman et al reported a case of a 55year-old woman undergoing treatment for CD20+ B-cell acute lymphoblastic leukemia. After recovering from COVID-19 pneumonia, she resumed chemotherapy, but soon lost COVID-19 antibodies, and her nasal PCR changed to positive, suffering from severe COVID-19 pneumonia. Given the short time from leukemia treatment to PCR positive, re-infection seems unlikely, and SARS-CoV-2 reactivation is a possible explanation. ${ }^{21}$ However, information on chemotherapy outcomes for cancer patients previously infected with COVID-19 is limited, and further 
work is needed to prospectively follow these topics in the long-term.

COVID-19 is also characterized by elevated cytokines, also known as cytokine storm, which manifests as uncontrolled systemic overproduction of cytokines, leading to a variety of pathologies. Cytokine storm is also called cytokine release syndrome (CRS). CRS is a non-antigen-specific toxicity, caused by high levels of immune activation, and is one of the main risks associated with cancer immunotherapy. ${ }^{22-24}$ Immunotherapy has become an emerging strategy for the treatment of cancer. Although immunotherapy has made significant progress, the clinical application of immunotherapy for cancer patients has some challenges in terms of safety and effectiveness, including autoimmune response, CRS, and vascular leakage syndrome. ${ }^{25}$ Chimeric antigen receptor $\mathrm{T}$ cell (CAR-T) therapy is an immunotherapy that specifically kills cancer cells by transforming the patient's own immune system, bringing a revolutionary leap in the treatment of some tumors. However, researchers have found that in the clinical application of CAR-T, CRS will be accompanied by significant side effects. Moreover, the more obvious the effect of CAR-T in killing tumor cells, the stronger the CRS. Excessive inflammation can cause fever, hypotension, heart problems, dyspnea, fatigue and nausea, and coagulopathy and other clinical manifestations. In severe cases, it will cause huge damage to body tissues and organs and even death. ${ }^{26}$ The incidence of CRS in patients receiving cancer immunotherapy varies with the type of immunotherapeutic agent. For most traditional monoclonal antibodies, the incidence of CRS is relatively low, and cancer immunotherapy involving $\mathrm{T}$ cells has a particularly high risk of $\mathrm{CRS}^{27}$ Therefore, when a cancer patient undergoing immunotherapy treatment is infected with COVID-19, the viral infection will aggravate the cytokine storm in the patient's body, which will increase the chance of severe clinical symptoms of COVID-19 in the cancer patient.

An investigation conducted by UK Lung Cancer Coalition (UKLCC) found that there are overlapping symptoms between lung cancer and COVID-19, such as persistent coughing and dyspnea, leading to the suspicion of SARS-CoV-2 infection in many early-stage lung cancer patients; they may self-quarantine without seeking medical screening and lose the opportunity for early diagnosis and treatment of cancer. $^{28}$ Furthermore, ground-glass opacity is the most common CT manifestation of COVID-19, especially in the early stage, which may cause confusion with the diagnosis of early lung cancer. ${ }^{29}$ In addition, because antiviral therapy disrupts the conventional treatment and the regular follow-up of cancer patients, the proportion of patients with advanced disease has increased. Therefore, the COVID19 epidemic has delayed the diagnosis and treatment of cancer.

\section{Patients with Cancer are Susceptible to Covid-I9}

Westblade et al's investigation found that the SARS-CoV -2 viral load is an independent predictor of the in-hospital mortality of patients with cancer. ${ }^{30}$ Among the cancer patients with COVID-19, the in-hospital mortality rates of patients with high, middle, and low viral load were $45.2 \%, 28 \%$, and $12.1 \%$, respectively $(\mathrm{p}=0.008) .{ }^{30}$ Patients with cancer have a weakened immune system and are often comorbid with type 2 diabetes, obesity, or cardiovascular disease, which leads to the overall poor health status and increases their susceptibility to SARSCoV-2. ${ }^{31-36}$ The burden of additional health loss varies in cancer patients with COVID-19, making it more challenging to predict disease progression and to manage disease symptoms in these patients. ${ }^{37}$ Furthermore, most anticancer drugs are cytotoxic, which inhibit the hematopoietic and immune systems and predispose the body to an immunosuppressive status, making cancer patients more susceptible to COVID-19 infection. ${ }^{38}$ Lee et al compared the data of cancer patient cohorts with or without COVID19 in the UK, and they found that, after multivariate adjustment of age- and gender-related clinical confounding factors, the condition of the patients with hematological malignancies was more serious after COVID-19 infection (odds ratio [OR] 1.57, 95\% CI 1.15-2.15; p < 0.0043 ) and the risk of death during hospitalization of the patients with hematological malignancies who have recently received chemotherapy were also increased after COVID-19 infection $^{39}$ (OR 2.09, 95\% CI 1.09-4.08; $\mathrm{p}=0.028$ ). In addition, Jee et al found that both the severity of hematological malignancies (HR, 1.90; 95\% CI, 1.30 to $2.80 ; \mathrm{P}<0.01)$ and the severity of lung cancer (HR, 2.00; 95\% CI, 1.20 to $3.30 ; \mathrm{P}=0.01$ ) were associated with the increase in COVID-19 severity. ${ }^{40}$ Kong et al found that lung adenocarcinoma patients are more likely to be infected with SARS-CoV-2 than lung squamous cell carcinoma patients, indicating that individuals 
with different malignant tumors may have distinct susceptibility to COVID-19. ${ }^{41}$ The clinical advisory team of UKLCC pointed out that if lung cancer patients are infected with COVID-19 after surgery, their mortality rates will increase by $40-50 \%$, and the risk of death of lung cancer may increase most severely among all cancers. ${ }^{28}$ COVID-19 patients with lung cancer patients are at higher risk of serious events than their counterparts without cancer.

Angiotensin-converting enzyme 2 (ACE2) is the receptor for SARS-CoV to enter the cell. ${ }^{42}$ SARS-CoV-2 is a new type of coronavirus of the same genus as SARS$\mathrm{CoV}$. ACE2 which is considered to be the SARS-CoV receptor, is also necessary SARS- CoV-2, and the expression of ACE2 in different tissues is closely related to the susceptibility and severity of viral infection. ${ }^{43,44}$ Ren et al used the GEPIA database to compare the mRNA expression of ACE2 in cancer and normal samples, and the results showed that almost all cancer tissues can express ACE2.$^{45}$ Chai et al found in biometrics analysis that ACE2 is overexpressed in colon adenocarcinoma (COAD), renal papillary cell carcinoma (KIRP), pancreatic adenocarcinoma (PAAD), rectal adenocarcinoma (READ), gastric adenocarcinoma (STAD) and lung adenocarcinoma (LUAD). ${ }^{46}$ The susceptibility of cancer patients to SARSCOV-2 may be closely related to the expression of ACE2. In addition, SARS-CoV-2 infection can reduce the activity of ACE2 receptors because the virus occupies these receptors, intervening in their normal function. In cancer patients with COVID-19, the ACE2 promoter is hypomethylated, and the level of hypomethylation is lower than that of their cancer counterparts without COVID19. ${ }^{47}$ DNA hypomethylation is related to gene activation during cancer progression. ${ }^{48}$ Hypomethylation can cause genome instability. The tumor tissues infected with SARSCoV-2 have a decrease in ACE2. ACE2 has a potential protective effect on cancer progression. The decrease in ACE2 leads to a decrease in immune infiltration and leads to disturbances in the tumor microenvironment, which

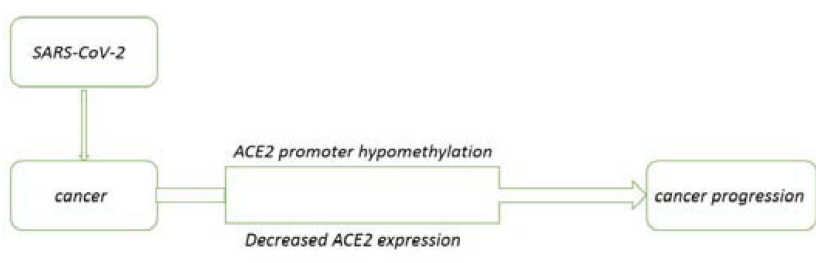

Figure I The mechanisms of SARS-CoV-2 in cancer progression. may worsen patients with SARS-CoV-2 tumors ${ }^{49,50}$ (Figure 1).

\section{The Impact of Antiviral Therapy on Cancer}

Viruses maintain their own survival by interfering with cell cycle regulatory components, reprogram the host epigenome, and promote metabolic pathways and epigenetic pathways, while cancer cells also choose the same pathways to proliferate or to invade other tissues. ${ }^{51}$ Therefore, some of the virus-suppression drugs may also be potential anticancer agents. At the present time, according to China's COVID-19 diagnosis and Treatment Plan (8th Trial Version), the treatment methods used in COVID-19 patients include: i) antiviral therapy (such as alpha-interferon, ribavirin, chloroquine phosphate, and arbidol hydrochloride), ii) immunotherapy (such as convalescent plasma, intravenous injection of COVID-19 human immunoglobulin, and tocilizumab), iii) glucocorticoid therapy, iv) some Chinese patent medicines, such as Qingfei Paidu Decoction, Lianhua Qingwen Capsules (granules), and so on. ${ }^{52}$

According to the regular press conference for COVID-19 held by the World Health Organization on October 16, dexamethasone was still considered as the only effective drug for severe COVID-19. ${ }^{53}$ Dexamethasone can inhibit the production of interleukin-6 (IL-6), the immune regulation driven by which is the main feature of SARS-CoV-2 infection. ${ }^{54}$ In the UK RECOVERY trial study, it was found that IL-6 level of the patients who received dexamethasone treatment dropped immediately, and that the risk of death for the patients receiving oxygen therapy with and without invasive mechanical ventilation were reduced by $35 \%$ and $20 \%$, respectively, after dexamethasone treatment. ${ }^{55}$ At the same time, IL- 6 was found to be the driving factor for tumorigenesis and anti-apoptotic signals as well as the key biomarker for the diagnosis, and prognosis evaluation of malignant tumor. ${ }^{56}$ Therefore, it will bring great significance to investigate not only the protective effect of IL-6 inhibitors against the inflammatory damage caused by COVID-19 but also their beneficial effects for cancer treatment. ${ }^{57}$ Ham et al studied the gastric cancer cell lines of MKN-1 and MKN-45 and found that the anti-IL-6 receptor monoclonal antibody tocilizumab can eliminate cancerassociated fibroblasts (CAF)-mediated inhibition of apoptosis. ${ }^{58}$ Compared with 5-fluorouracil treatment alone, combination treatment with tocilizumab and 5-fluorouracil significantly reduced the cancer weight of CAF-mixed xenograft mice. At the present time, the interaction rate between 
anti-cancer drugs and anti-COVID-19 drugs is relatively modest, ${ }^{38}$ and the therapeutic effect of anti-COVID-19 drugs on cancer patients needs to be explored according to numerous experimental and clinical data.

Most recently, Challenor et al reported the alleviation of lymphadenopathy in a patient with Epstein-Barr virus (EBV)-positive classical Hodgkin lymphoma after his infection with SARS-CoV-2. No corticosteroid or immunochemotherapy was given, and the underlying mechanism was probably attributed to the anti-tumor immune response induced by SARS-CoV-2 infection. This suggests a potential association between anti-viral and anti-tumor treatments and sheds light on the immunotherapy of malignant disease. ${ }^{59}$

\section{Cancer Research and Clinical Practice in Covid- 19 Pandemic: Impact and Countermeasures}

\section{Impact}

The COVID-19 outbreak has affected the progress of cancer-related research. As long as there is a high proportion of COVID-19 cases, even if the patients with cancer return to the pre-pandemic status, they are unlikely to participate in cancer clinical trials. ${ }^{60}$ Due to the COVID19 pandemic, the access of scientific researchers and students to the laboratories is restricted, many of the scientific research projects are temporarily suspended, and field trips have to be interrupted. ${ }^{61}$ Some mice in the research stage of cancer models have to be euthanized, years of hard work may be ruined, and many studies have no choice but to be interrupted. ${ }^{62}$ Global data shows some of the trends seen in three months of interrupted trials by treatment area. Since April 2020, the overall share of oncology has begun to decline, from $33.3 \%$ to $30.1 \%{ }^{70}$ Since many patients have had severe COVID-19, the potential complications and damage to the body caused by the disease may have some long-lasting impacts even after their recovery. COVID-19 has become a long-term problem.

\section{Countermeasures}

To improve cancer research and clinical practice during the COVID-19 pandemic, some major cancer research centers have decided to continue their trials and consider specific preparations. For instance, the researchers at the University of Arizona Cancer Center have limited their trials to those that are potentially life-prolonging. At the Perlmutter Cancer Center in New York, researchers have stratified the trials, putting Phase 2 or 3 clinical trials as a priority and halting early-phase ones. The researchers at the Yale Cancer Center in New Haven have followed a similar strategy by continuing the ongoing trials and halting the beginning ones. ${ }^{63}$ During the COVID-19 epidemic period, the management of drug shortages is complicated, especially in the field of oncology. The special situation of oncology requires advanced strategies to deal with this problem. In 2018, the American Association of Health System Pharmacists (ASHP) put forward recommendations for managing drug shortages, including the rational allocation of drug resources, and the search and approval of alternative drugs or therapeutic equivalents. By following this guideline, health care institutions can mitigate the impact of the pandemic on the provision of necessary medicines and reduce its adverse effects on patient care. $^{64}$ The American Society of Clinical Oncology (ASCO) organizes recommendations on cancer care as well, such as ensuring the fairness of patients' access to high-quality cancer care and solving the health problems of cancer patients. ${ }^{65}$ During the peak period of COVID-19, the medical resources should be arranged reasonably between the emergency treatment of COVID19 and the regular care of cancer patients. In order to reduce the burden of cancer caused by COVID-19, it is encouraged to take any measures that can be treated at home to minimize the hospitalization of cancer patients. Online consultation channels should be open for cancer patients to provide medication guidance and symptom management. For patients who require surgery, a comprehensive evaluation should be carried out to determine the appropriate timing of treatment. ${ }^{66}$ As patients with cancer are susceptible to COVID-19, the risk-benefit ratio of palliative and adjuvant treatments should also be considered. Butler et al have proposed some new methods for the treatment of cancer in the pandemics of COVID-19, such as running media promotion to improve the acceptance of cancer screening, encouraging patients to seek medical help when potential cancer symptoms occur, investing in technology and infrastructure to facilitate virtual consultations, holding multidisciplinary team meetings and other innovations, and benchmarking international and regional cancer outcomes in the context of COVID-19. ${ }^{67}$ The American Association for Cancer Research (AACR) is trying to collect and analyze data from the patients with cancer and COVID-19 through the Association for Malignant Cancer (CCC19) and to put forward the proposal for 
reducing the risk of cancer patients with further research. $^{68}$

\section{Conclusion}

At present, the epidemic is not over, and the world is still invaded by COVID-19. Although the clinical features of COVID-19 have been described in detail, the molecular and cellular mechanisms of its pathogenesis are still elusive. Will COVID-19 change the way cancer grows or spreads? How long will COVID-19 harm the quality of life of cancer patients? These issues are still waiting to be resolved. At the same time, researchers have discovered the risk factors and markers of serious diseases related to COVID-19 from cancer patients, and conducted detailed and in-depth evaluation of the biomarkers that are severely related to the results of severe COVID-19. It will enable us in the future to identify people who need more aggressive treatment or preventive measures. With the inoculation of the new crown vaccine, the incidence of COVID-19 has been significantly reduced. However, as an infectious disease, COVID-19 is likely to continue to exist, and it will also have a continuous impact on the diagnosis, treatment and prognosis of tumors. The clinic also needs to pay attention to the mutual influence between the two. Countermeasures against the impact of the COVID-19 pandemic on cancer should be seriously considered to reduce the mortality rate of cancer caused by the COVID-19 pandemic and to improve the quality of life of cancer patients. ${ }^{69}$

\section{Acknowledgment}

This work was supported by the emergency project of Liaoning provincial science and technology department, No. 2020JH2/10300003.

\section{Disclosure}

The authors declare no conflicts of interest in this work.

\section{References}

1. Pascarella G, Strumia A, Piliego C, et al. COVID-19 diagnosis and management: a comprehensive review. J Intern Med. 2020;288 (2):192-206. doi:10.1111/joim.13091

2. Fang Z, Fang Y, Kang W, et al. Clinical characteristics of Coronavirus Disease 2019 (COVID-19): an updated systematic review. medRxiv. 2020. Available from: https://www.medrxiv.org/content/10.1101/2020. 03.07.20032573v2. Accessed September 8, 2021.

3. Gupta A, Madhavan MV, Sehgal K, et al. Extrapulmonary manifestations of COVID-19. Nat Med. 2020;26(7):1017-1032. doi:10.1038/ s41591-020-0968-3
4. National Health Commission of the People's Republic of China. COVID-19 is Included in the Management of Legal Infectious Diseases; January 20, 2020.

5. WHO. COVID-19 Weekly Epidemiological Update; January 3, 2020.

6. Disis ML. Oncology and COVID-19. JAMA. 2020;324(12):11 41-1142. doi:10.1001/jama.2020.16945

7. Jones D, Neal RD, Duffy SRG, Scott SE, Whitaker KL, Brain K. Impact of the COVID-19 pandemic on the symptomatic diagnosis of cancer: the view from primary care. Lancet Oncol. 2020;21 (6):748-750. doi:10.1016/S1470-2045(20)30242-4

8. Tagliamento M, Lambertini M, Genova C, et al. Call for ensuring cancer care continuity during COVID-19 pandemic. ESMO Open. 2020;5(3):e000783. doi:10.1136/esmoopen-2020-000783

9. Islam JY, Camacho-Rivera M, Vidot DC. Examining COVID-19 preventive behaviors among cancer survivors in the United States: an analysis of the COVID-19 impact survey. Cancer Epidemiol Biomarkers Prev. 2020;29(12):2583-2590. doi:10.1158/1055-9965.EPI-20-0801

10. Challinor JM, Alqudimat MR, Teixeira TOA, Oldenmenger WH. Oncology nursing workforce: challenges, solutions, and future strategies. Lancet Oncol. 2020;21(12):e564-e574. doi:10.1016/ S1470-2045(20)30605-7

11. Burki TK. Burnout among cancer professionals during COVID-19. Lancet Oncol. 2020;21(11):1402. doi:10.1016/S1470-2045(20)30584-2

12. Bakouny Z, Paciotti M, Schmidt AL, Lipsitz SR, Choueiri TK, Trinh Q-D. Cancer screening tests and cancer diagnoses during the COVID-19 pandemic. JAMA Oncol. 2021;7(3):458. doi:10.1001/ jamaoncol.2020.7600

13. Maringe C, Spicer J, Morris M, et al. The impact of the COVID-19 pandemic on cancer deaths due to delays in diagnosis in England, UK: a national, population-based, modelling study. Lancet Oncol. 2020;21(8):1023-1034. doi:10.1016/S1470-2045(20)30388-0

14. Liang W, Guan W, Chen R, et al. Cancer patients in SARS-CoV-2 infection: a nationwide analysis in China. Lancet Oncol. 2020;21 (3):335-337. doi:10.1016/S1470-2045(20)30096-6

15. Johnson BA, Waddimba AC, Ogola GO, Fleshman JW Jr, Preskitt JT. A systematic review and meta-analysis of surgery delays and survival in breast, lung and colon cancers: implication for surgical triage during the COVID-19 pandemic. Am J Surg. 2020(2021):331-318.

16. Sud A, Jones ME, Broggio J, et al. Collateral damage: the impact on outcomes from cancer surgery of the COVID-19 pandemic. Ann Oncol. 2020;31(8):1065-1074.10. doi:10.1016/j.annonc.2020.05.009

17. Riemann S, Speck I, Gerstacker K, Becker C, Knopf A. Collateral damage of the COVID-19 pandemic: an alarming decline in critical procedures in otorhinolaryngology in a German university hospital. Eur Arch Otorhinolaryngol. 2020;278(9):3417-3423.

18. Zhang L, Zhu F, Xie L, et al. Clinical characteristics of COVID-19infected cancer patients: a retrospective case study in three hospitals within Wuhan, China. Ann Oncol. 2020;31(7):894-901. doi:10.1016/ j.annonc.2020.03.296

19. Al-Shamsi HO, Alhazzani W, Alhuraiji A, et al. A practical approach to the management of cancer patients during the novel Coronavirus Disease 2019 (COVID-19) pandemic: an International Collaborative Group. Oncologist. 2020;25(6):e936-e945. doi:10.1634/theoncologist.2020-0213

20. National Comprehensive Cancer Network. Coronavirus Disease 2019 (COVID-19) resources for the cancer care community. (2020 Version 1). Available from: www.ncen.org/covid-19/default.aspx. Accessed August 25, 2021.

21. Lancman G, Mascarenhas J, Bar-Natan M. Severe COVID-19 virus reactivation following treatment for B cell acute lymphoblastic leukemia. J Hematol Oncol. 2020;13(1):131. doi:10.1186/s13045020-00968-1

22. Cameron BJ, Gerry AB, Dukes $\mathrm{J}$, et al. Identification of a titin-derived HLA-A1-presented peptide as a cross-reactive target for engineered MAGE A3-directed T cells. Sci Transl Med. 2013;5 (197):197ra103. doi:10.1126/scitranslmed.3006034 
23. Linette GP, Stadtmauer EA, Maus MV, et al. Cardiovascular toxicity and titin cross-reactivity of affinity-enhanced $\mathrm{T}$ cells in myeloma and melanoma. Blood. 2013;122(6):863-871. doi:10.1182/blood-201303-490565

24. Morgan RA, Chinnasamy N, Abate-Daga D, et al. Cancer regression and neurological toxicity following anti-MAGE-A3 TCR gene therapy. J Immunother. 2013;36(2):133-151. doi:10.1097/CJI.0b01 3e3182829903

25. Zhao Z, Zheng L, Chen W, Weng W, Song J, Ji J. Delivery strategies of cancer immunotherapy: recent advances and future perspectives. J Hematol Oncol. 2019;12(1):126. doi:10.1186/s13045-019-0817-3

26. Bonifant CL, Jackson HJ, Brentjens RJ, Curran KJ. Toxicity and management in CAR T-cell therapy. Mol Ther Oncolytics. 2016;3:16011. doi:10.1038/mto.2016.11

27. Lee DW, Gardner R, Porter DL, et al. Current concepts in the diagnosis and management of cytokine release syndrome. Blood. 2014;124(2):188-195. doi:10.1182/blood-2014-05-552729

28. Gourd E. Lung cancer control in the UK hit badly by COVID-19 pandemic. Lancet Oncol. 2020;21(12):1559. doi:10.1016/S14702045(20)30691-4

29. Zhang YJ, Yang WJ, Liu D, et al. COVID-19 and early-stage lung cancer both featuring ground-glass opacities: a propensity score-matched study. Transl Lung Cancer Res. 2020;9 (4):1516-1527. doi:10.21037/tlcr-20-892

30. Westblade LF, Brar G, Pinheiro LC, et al. SARS-CoV-2 viral load predicts mortality in patients with and without cancer who are hospitalized with COVID-19. Cancer Cell. 2020;38(5):661-671.e2. doi:10.1016/j.ccell.2020.09.007

31. Schreiber RD, Old LJ, Smyth MJ. Cancer immunoediting: integrating immunity's roles in cancer suppression and promotion. Science. 2011;331(6024):1565-1570. doi:10.1126/science. 1203486

32. Giovannucci E, Harlan DM, Archer MC, et al. Diabetes and cancer: a consensus report. Diabetes Care. 2010;33(7):1674-1685. doi:10.2337/dc10-0666

33. Kitsis RN, Riquelme JA, Lavandero S. Heart disease and cancer: are the two killers colluding? Circulation. 2018;138(7):692-695. doi:10.1161/CIRCULATIONAHA.118.033907

34. Chen W, Zheng R, Baade PD, et al. Cancer statistics in China, 2015. CA Cancer J Clin. 2016;66(2):115-132. doi:10.3322/caac.21338

35. Lauby-Secretan B, Scoccianti C, Loomis D, Grosse Y, Bianchini F, Straif K; International Agency for Research on Cancer Handbook Working Group. Body fatness and cancer-viewpoint of the IARC Working Group. N Engl J Med. 2016;375(8):794-798. doi:10.1056/ NEJMsr1606602

36. American Cancer Society. Why People with Cancer are More Likely to Get Infections; March 2020. Accessed August 14, 2020.

37. Wang Q, Berger NA, Xu R. Analyses of risk, racial disparity, and outcomes among US patients with cancer and COVID-19 infection. JAMA Oncol. 2020;10:e206178.

38. Di Lorenzo G, Di Trolio R, Kozlakidis Z, et al. COVID 19 therapies and anti-cancer drugs: a systematic review of recent literature. Crit Rev Oncol Hematol. 2020;152:102991. doi:10.1016/j.critrevonc.20 20.102991

39. Lee LYW, Cazier JB, Starkey T, Briggs SEW, Arnold R, Bisht V. COVID-19 prevalence and mortality in patients with cancer and the effect of primary tumour subtype and patient demographics: a prospective cohort study. Lancet Oncol. 2020;21(10):1309-1316. doi:10.1016/S1470-2045(20)30442-3

40. Jee J, Foote MB, Lumish M, et al. Chemotherapy and COVID-19 outcomes in patients with cancer. J Clin Oncol. 2020;38 (30):3538-3546. doi:10.1200/JCO.20.01307

41. Kong Q, Xiang Z, Wu Y, Gu Y, Guo J, Geng F. Analysis of the susceptibility of lung cancer patients to SARS-CoV-2 infection. Mol Cancer. 2020;19(1):80. doi:10.1186/s12943-020-01209-2
42. Tutuncuoglu B, Cakir M, Batra J, et al. The landscape of human cancer proteins targeted by SARS-CoV-2. Cancer Discov. 2020;10 (7):916-921. doi:10.1158/2159-8290.CD-20-0559

43. Li W, Moore MJ, Vasilieva N, et al. Angiotensin-converting enzyme 2 is a functional receptor for the SARS coronavirus. Nature. 2003;426(6965):450-454. doi:10.1038/nature02145

44. Hou Y, Peng C, Yu M, et al. Angiotensin-converting enzyme 2 (ACE2) proteins of different bat species confer variable susceptibility to SARS-CoV entry. Arch Virol. 2010;155(10):1563-1569. doi:10.10 07/s00705-010-0729-6

45. Hattermann K, Müller MA, Nitsche A, Wendt S, Donoso Mantke O, Niedrig M. Susceptibility of different eukaryotic cell lines to SARS-coronavirus. Arch Virol. 2005;150(5):1023-1031. doi:10.10 07/s00705-004-0461-1

46. Ren P, Gong C, Ma S. Evaluation of COVID-19 based on ACE2 expression in normal and cancer patients. Open Med. 2020;15 (1):613-622. doi:10.1515/med-2020-0208

47. Chai P, Yu J, Ge S, Jia R, Fan X. Genetic alteration, RNA expression, and DNA methylation profiling of coronavirus disease 2019 (COVID-19) receptor ACE2 in malignancies: a pan-cancer analysis. J Hematol Oncol. 2020;13(1):43. doi:10.1186/s13045-020-00883-5

48. Yang J, Li H, Hu S, Zhou Y. ACE2 correlated with immune infiltration serves as a prognostic biomarker in endometrial carcinoma and renal papillary cell carcinoma: implication for COVID-19. Aging. 2020;12(8):6518-6535. doi:10.18632/aging.103100

49. Ehrlich M. DNA hypomethylation in cancer cells. Epigenomics. 2009;1(2):239-259. doi:10.2217/epi.09.33

50. Wang XS. Angiotensin-converting enzyme 2 connects COVID-19 with cancer and cancer immunotherapy. World $J$ Gastrointest Oncol. 2021;13(3):157-160. doi:10.4251/wjgo.v13.i3.157

51. Pathania AS, Prathipati P, Abdul BA, et al. COVID-19 and cancer comorbidity: therapeutic opportunities and challenges. Theranostics. 2021;11(2):731-753. doi:10.7150/thno.51471

52. National Health Commission of the People's Republic of China. Diagnosis and Treatment Plan of COVID-19 (8th Trial Version); August 19, 2020.

53. Garassino MC, Whisenant JG, Huang LC. COVID-19 in patients with thoracic malignancies (TERAVOLT): first results of an international, registry-based, cohort study. Lancet Oncol. 2020;21 (7):914-922. doi:10.1016/S1470-2045(20)30314-4

54. Galluzzi L, Buque A, Kepp O, Zitvogel L, Kroemer G. Immunological effects of conventional chemotherapy and targeted anticancer agents. Cancer Cell. 2015;28(6):690-714. doi:10.1016/j. ccell.2015.10.012

55. Dexamethasone for COVID-19. preliminary findings. Drug Ther Bull. 2020;58(9):133. doi:10.1136/dtb.2020.000045

56. Vargas AJ, Harris CC. Biomarker development in the precision medicine era: lung cancer as a case study. Nat Rev Cancer. 2016;16 (8):525-537. doi:10.1038/nrc.2016.56

57. Turnquist C, Ryan BM, Horikawa I, Harris BT, Harris CC. Cytokine storms in cancer and COVID-19. Cancer Cell. 2020;38(5):598-601. doi:10.1016/j.ccell.2020.09.019

58. Ham IH, Oh HJ, Jin H, et al. Targeting interleukin-6 as a strategy to overcome stroma-induced resistance to chemotherapy in gastric cancer. Mol Cancer. 2019;18(1):68. doi:10.1186/s12943-019-0972-8

59. Challenor S, Tucker D. SARS-CoV-2-induced remission of Hodgkin lymphoma. Br J Haematol. 2021;192(3):415. doi:10.1111/bjh.17116

60. Fleury ME, Farner AM, Unger JM. Association of the COVID-19 outbreak with patient willingness to enroll in cancer clinical trials. JAMA Oncol. 2021;7(1):131-132. doi:10.1001/jamaoncol.2020.5748

61. Sheridan K. U.S. biotechs fear the coronavirus outbreak will delay their China-based research. Available from: https://www.statnews. com/2020/02/11/biotechs-fear-coronavirus-will-delay-china-basedresearch/. Accessed February 11, 2020. 
62. Burki TK. Cuts in cancer research funding due to COVID-19. Lancet Oncol. 2021;22(1):e6. doi:10.1016/S1470-2045(20)30749-X

63. Ribas A, Leng G.Clinical research slows as COVID-19 surges. Cancer Discov. 2020;10(5):630. doi:10.1158/2159-8290.

64. Fadavi P, Houshyari M, Yousefi Kashi AS, et al. Review on the oncology practice in the midst of COVID-19 crisis: the challenges and solutions. Asian Pac J Cancer Prev. 2021;22(1):19-24. doi:10. 31557/APJCP.2021.22.1.19

65. Pennell NA, Dillmon M, Levit LA, et al. American society of clinical oncology road to recovery report: learning from the COVID-19 experience to improve clinical research and cancer care. J Clin Oncol. 2021;39(2):155-169. doi:10.1200/JCO.20.02953

66. Tzeng CD, Teshome M, Katz MHG, et al. Cancer surgery scheduling during and after the COVID-19 first wave: the MD Anderson cancer center experience. Ann Surg. 2020;272(2):e106-e111. doi:10.1097/ SLA.0000000000004092
67. Butler J, Finley C, Norell CH, et al. New approaches to cancer care in a COVID-19 world. Lancet Oncol. 2020;21(7):e339-e340. doi:10.1016/S1470-2045(20)30340-5

68. AACR Board of Directors. AACR calls on congress to take immediate action against COVID-19 and protect patients with cancer during the pandemic. Cancer Discov. 2020;10(6):771-774. doi:10.1158/ 2159-8290.CD-20-0449

69. Gyawali B, Poudyal BS, Eisenhauer EA. Covid-19 pandemic — an opportunity to reduce and eliminate low-value practices in oncology? JAMA Oncol. 2020;6(11):1693. doi:10.1001/jamaoncol.2020.2404

70. GlobalData Healthcare. Clinical trial disruption due to Covid-19 has begun to slow. Available from: https://www.clinicaltrialsarena.com/com ment/clinical-trial-disruption-slowing/. Accessed August 30, 2021.

\section{Publish your work in this journal}

Infection and Drug Resistance is an international, peer-reviewed openaccess journal that focuses on the optimal treatment of infection (bacterial, fungal and viral) and the development and institution of preventive strategies to minimize the development and spread of resistance. The journal is specifically concerned with the epidemiology of antibiotic resistance and the mechanisms of resistance development and diffusion in both hospitals and the community. The manuscript management system is completely online and includes a very quick and fair peerreview system, which is all easy to use. Visit http://www.dovepress.com/ testimonials.php to read real quotes from published authors. 